\title{
MONOTONICITY OF SEQUENCES INVOLVING GENERALIZED CONVEXITY FUNCTION AND SEQUENCES
}

\author{
NGUYEN NGOC HUE AND DUONG QUOC HUY
}

\begin{abstract}
In this paper, by using the theory of generalized convexity functions we introduce and prove monotonicity of sequences of the forms

$$
\begin{aligned}
& \left\{\left(\prod_{k=1}^{n} f\left(\frac{a_{k}}{a_{n}}\right)\right)^{1 / n}\right\}, \quad\left\{\left(\prod_{k=1}^{n} f\left(\frac{\varphi(k)}{\varphi(n)}\right)\right)^{1 / \varphi(n)}\right\}, \\
& \left\{\frac{1}{n} \sum_{k=1}^{n} f\left(\frac{a_{n}}{a_{k}}\right)\right\} \quad \text { or } \quad\left\{\frac{1}{\varphi(n)} \sum_{k=1}^{n} f\left(\frac{\varphi(n)}{\varphi(k)}\right)\right\},
\end{aligned}
$$

where $f$ belongs to the classes of $A G$-convex (concave), $H A$-convex (concave), or $H G$ convex (concave) functions defined on suitable intervals, $\left\{a_{n}\right\}$ is a given sequence and $\varphi$ is a given function that satisfy some preset conditions. As a consequence, we obtain some generalizations of Alzer type inequalities.
\end{abstract}

\section{Introduction}

Let $f$ be a real-valued function defined on $[a, b] \subset \mathbb{R}$. The function $f$ is called convex if

$$
f(\lambda x+(1-\lambda) y) \leq \lambda f(x)+(1-\lambda) f(y) .
$$

for all $x, y \in[a, b]$ and $\lambda \in[0,1]$. If (1.1) is strict for all $x \neq y$ and $\lambda \in(0,1)$, then $f$ is said to be strictly convex. If the inequality in (1.1) is reversed, then $f$ is said to be concave. If the inequality (1.1) is reversed and strict for all $x \neq y$ and $\lambda \in(0,1)$, then $\mathrm{f}$ is said to be strictly concave.

Suppose that $I$ is a subinterval of $(0, \infty)$. A function $f: I \rightarrow(0, \infty)$ is called multiplicatively convex if for all $x, y \in I$ and $\lambda \in[0,1]$,

$$
f\left(x^{\lambda} y^{1-\lambda}\right) \leq f(x)^{\lambda} f(y)^{1-\lambda} .
$$

Received March 18, 2014, accepted April 9, 2014.

2010 Mathematics Subject Classification. 26A51, 26D15.

Key words and phrases. Alzer's inequality, monotonicity, generalized convexity, sequences.

Corresponding author: Nguyen Ngoc Hue. 
If (1.2) is strict for all $x \neq y$ and $\lambda \in(0,1)$, then $f$ is said to be strictly multiplicatively convex. If the inequality in (1.2) is reversed, then $f$ is said to be multiplicatively concave. If inequality (1.2) is reversed and strict for all $x \neq y$ and $\lambda \in(0,1)$, then $f$ is said to be strictly multiplicatively concave.

In [3], F. Qi and B.-N. Guo proved the following theorems:

Theorem 1.1 ([3]). Let $f$ be an increasing, convex (concave, respectively) function defined on $[0,1],\left\{a_{n}\right\}$ an increasing, positive sequence such that $\left\{n\left(\frac{a_{n}}{a_{n+1}}-1\right)\right\}$ decreases (the sequence $\left\{n\left(\frac{a_{n+1}}{a_{n}}-\right.\right.$ 1)\} increses, respectively), then

$$
\frac{1}{n} \sum_{k=1}^{n} f\left(\frac{a_{k}}{a_{n}}\right) \geq \frac{1}{n+1} \sum_{k=1}^{n+1} f\left(\frac{a_{k}}{a_{n+1}}\right) \geq \int_{0}^{1} f(x) d x
$$

and

Theorem 1.2 ([3]). Let $f$ be an increasing convex (or concave) positive function defined on $[0,1], \varphi$ be an increasing convex positive function defined on $(0, \infty)$ such that $\left\{\varphi(k)\left(\frac{\varphi(k)}{\varphi(k+1)}-1\right)\right\}$ decreases, then

$$
\frac{1}{\varphi(n)} \sum_{k=1}^{n} f\left(\frac{\varphi(k)}{\varphi(n)}\right) \geq \frac{1}{\varphi(n+1)} \sum_{k=1}^{n+1} f\left(\frac{\varphi(k)}{\varphi(n+1)}\right) .
$$

Jiding Liao and Kaizhong Guan [2] proved the following theorems:

Theorem 1.3 ([2]). Let $f$ be a positive function defined in $(0,1]$. Suppose that $\left\{a_{n}\right\}$ is an increasing positive sequence such that the sequence $\left\{\left(\frac{a_{n+1}}{a_{n}}\right)^{n}\right\}$ increases.

(1) If $f$ is an increasing and multiplicatively convex (concave) function, then

$$
\left(\prod_{k=1}^{n} f\left(\frac{a_{k}}{a_{n}}\right)\right)^{1 / n} \geq\left(\prod_{k=1}^{n+1} f\left(\frac{a_{k}}{a_{n+1}}\right)\right)^{1 /(n+1)} .
$$

(2) If $f$ is an decreasing and multiplicatively convex (concave) function, then

$$
\left(\prod_{k=1}^{n} f\left(\frac{a_{k}}{a_{n}}\right)\right)^{1 / n} \leq\left(\prod_{k=1}^{n+1} f\left(\frac{a_{k}}{a_{n+1}}\right)\right)^{1 /(n+1)} .
$$

and

Theorem $1.4([2])$. Let $f:(0,1] \rightarrow[1,+\infty)$ be a real-valued function and $\left\{a_{n}\right\}$ an increasing positive sequence such that the sequence $\left\{\left(\frac{a_{n+1}}{a_{n}}\right)^{a_{n}}\right\}$ increases. Then the following statements are valid. 
(1) If $f$ is an increasing and multiplicatively convex (concave) function and $\left\{a_{n}\right\}$ is convex sequence, i.e., $a_{n-1}+a_{n+1} \geq 2 a_{n},(n=1,2, \ldots)$ where $a_{0}=0$, then

$$
\left(\prod_{k=1}^{n} f\left(\frac{a_{k}}{a_{n}}\right)\right)^{1 / a_{n}} \geq\left(\prod_{k=1}^{n+1} f\left(\frac{a_{k}}{a_{n+1}}\right)\right)^{1 / a_{n+1}} .
$$

(2) If $f$ is an decreasing and multiplicatively convex (concave) function and $\left\{a_{n}\right\}$ is concave sequence, i.e., $a_{n-1}+a_{n+1} \leq 2 a_{n},(n=1,2, \ldots)$ where $a_{0}=0$, then

$$
\left(\prod_{k=1}^{n} f\left(\frac{a_{k}}{a_{n}}\right)\right)^{1 / a_{n}} \leq\left(\prod_{k=1}^{n+1} f\left(\frac{a_{k}}{a_{n+1}}\right)\right)^{1 / a_{n+1}} .
$$

The above results are valid for the convex (concave) function and multiplicatively convex (concave) function. In [1], the authors introduced the class of mean function and generalized convexity. The class related directly to convex (concave) function.

Definition 1.1 ([1]). A function $M:(0, \infty) \times(0, \infty) \rightarrow(0, \infty)$ is called a mean function if

(1) $M(x, y)=M(y, x)$;

(2) $M(x, x)=x$;

(3) $x<M(x, y)<y$, whenever $x<y$;

(4) $M(a x, a y)=a M(x, y)$ for all $a>0$.

Some familiar mean functions such as Arithmetic Mean, Geometric Mean, Harmonic Mean, Logarithmic Mean, Identric Mean and denoted by $A, G, H, L, I$, respectively. For details concerning mean functions $A, G, H, L, I$ we refer to the papers [1] and [5].

Definition $1.2([1])$. Let $f: I \rightarrow(0, \infty)$ be continuous, where $I$ is a subinterval of $(0, \infty)$. Let $M$ and $N$ be any two mean functions. We say $f$ is $M N$-convex (concave) if

$$
f(M(x, y)) \leq(\geq) N(f(x), f(y))
$$

for all $x, y \in I$.

From Definition 1.2, the inequalities (1.1) and (1.2) can be rewritten under the simple forms

$$
f(A(x, y)) \leq A(f(x), f(y)) \text { and } f(G(x, y)) \leq G(f(x), f(y)) .
$$

More precisely, $f$ is $A A$-convex for the first case and $G G$-convex for the second case.

Our main purpose of this paper is to present some inequalities which are similar to the results in [2] and [3] for some generalized convexity functions such as $A G$-convex (concave), $H A$-convex (concave) and $H G$-convex (concave). 


\section{The main results}

In this section, we investigate the monotonicity of some sequences involving $A G, H A$, $H G$ - convex (concave) function and convex sequence.

Theorem 2.1. Let $f$ be an increasing, AG-convex (concave, respectively) function defined on $(0,1]$.

(1) If $\left\{a_{n}\right\}$ is an increasing, positive sequence such that $\left\{n\left(\frac{a_{n}}{a_{n+1}}-1\right)\right\}$ decreases (the sequence $\left\{n\left(\frac{a_{n+1}}{a_{n}}-1\right)\right\}$ increses, respectively), then

$$
\left(\prod_{k=1}^{n} f\left(\frac{a_{k}}{a_{n}}\right)\right)^{1 / n} \geq\left(\prod_{k=1}^{n+1} f\left(\frac{a_{k}}{a_{n+1}}\right)\right)^{1 /(n+1)} .
$$

(2) If $\varphi$ is an increasing convex positive function defined on $(0, \infty)$ such that $\left\{\varphi(k)\left(\frac{\varphi(k)}{\varphi(k+1)}-\right.\right.$ 1)\} decreases, then

$$
\left(\prod_{k=1}^{n} f\left(\frac{\varphi(k)}{\varphi(n)}\right)\right)^{1 / \varphi(n)} \geq\left(\prod_{k=1}^{n+1} f\left(\frac{\varphi(k)}{\varphi(n+1)}\right)\right)^{1 / \varphi(n+1)} .
$$

Proof. Here we only give the proof of the $A G$-convex, since that the $A G$-concave is similar and we omit it.

By Theorem 2.4 in [1], the function $f$ is $A G$-convex (concave) if and only if $\ln f$ is convex (concave). Obviously, $\ln f$ increases by the increase of $f$. Hence, applying Theorem 1.1 for $\ln f$, we have

$$
\frac{1}{n} \sum_{k=1}^{n} \ln f\left(\frac{a_{k}}{a_{n}}\right) \geq \frac{1}{n+1} \sum_{k=1}^{n+1} \ln f\left(\frac{a_{k}}{a_{n+1}}\right) .
$$

It is equivalent to

$$
\ln \prod_{k=1}^{n} f\left(\frac{a_{k}}{a_{n}}\right)^{1 / n} \geq \ln \prod_{k=1}^{n+1} f\left(\frac{a_{k}}{a_{n+1}}\right)^{1 /(n+1)} \Leftrightarrow\left(\prod_{k=1}^{n} f\left(\frac{a_{k}}{a_{n}}\right)\right)^{1 / n} \geq\left(\prod_{k=1}^{n+1} f\left(\frac{a_{k}}{a_{n+1}}\right)\right)^{1 /(n+1)} .
$$

So, the proof of (2.1) is complete.

Analogously, if applying Theorem 1.2 for $\ln f$, then

$$
\frac{1}{\varphi(n)} \sum_{k=1}^{n} \ln f\left(\frac{\varphi(k)}{\varphi(n)}\right) \geq \frac{1}{\varphi(n+1)} \sum_{k=1}^{n+1} \ln f\left(\frac{\varphi(k)}{\varphi(n+1)}\right) .
$$

Equivalently,

$\ln \prod_{k=1}^{n} f\left(\frac{\varphi(k)}{\varphi(n)}\right)^{1 / \varphi(n)} \geq \ln \prod_{k=1}^{n+1} f\left(\frac{\varphi(k)}{\varphi(n)}\right)^{1 / \varphi(n+1)} \Leftrightarrow\left(\prod_{k=1}^{n} f\left(\frac{\varphi(k)}{\varphi(n)}\right)\right)^{1 / \varphi(n)} \geq\left(\prod_{k=1}^{n+1} f\left(\frac{\varphi(k)}{\varphi(n+1)}\right)\right)^{1 / \varphi(n+1)}$.

Hence, the inequality (2.2) is completely proved. 
Theorem 2.2. Let $f$ be a decreasing, HA-convex (concave, respectively) function defined on $[1,+\infty)$.

(1) If $\left\{a_{n}\right\}$ an increasing, positive sequence such that $\left\{n\left(\frac{a_{n}}{a_{n+1}}-1\right)\right\}$ decreases (the sequence $\left\{n\left(\frac{a_{n+1}}{a_{n}}-1\right)\right\}$ increses, respectively), then

$$
\frac{1}{n} \sum_{k=1}^{n} f\left(\frac{a_{n}}{a_{k}}\right) \geq \frac{1}{n+1} \sum_{k=1}^{n+1} f\left(\frac{a_{n+1}}{a_{k}}\right) .
$$

(2) If $\varphi$ be an increasing convex positive function defined on $(0, \infty)$ such that $\left\{\varphi(k)\left(\frac{\varphi(k)}{\varphi(k+1)}-\right.\right.$ 1)\} decreases, then

$$
\frac{1}{\varphi(n)} \sum_{k=1}^{n} f\left(\frac{\varphi(n)}{\varphi(k)}\right) \geq \frac{1}{\varphi(n+1)} \sum_{k=1}^{n+1} f\left(\frac{\varphi(n+1)}{\varphi(k)}\right) .
$$

Proof. Here we only give the proof of (2), since that (1) is similar and we omit it.

By Theorem 2.4 in [1], the function $f$ is $H A$-convex (concave) if and only if $f(1 / x)$ is convex (concave). It's easy to see that $g(x):=f(1 / x)$ increases by the decrease of $f$. Hence, applying Theorem 1.2 for $g$, we have

$$
\frac{1}{\varphi(n)} \sum_{k=1}^{n} g\left(\frac{\varphi(k)}{\varphi(n)}\right) \geq \frac{1}{\varphi(n+1)} \sum_{k=1}^{n+1} g\left(\frac{\varphi(k)}{\varphi(n+1)}\right) .
$$

Noting that, in the above inequality, $g\left(\frac{\varphi(k)}{\varphi(n)}\right)=f\left(\frac{\varphi(n)}{\varphi(k)}\right)$ for all $k=1,2, \ldots, n$ and $g\left(\frac{\varphi(k)}{\varphi(n+1)}\right)=$ $f\left(\frac{\varphi(n+1)}{\varphi(k)}\right)$ for all $k=1,2, \ldots, n+1$, and so the proof of the inequality (2.4) is complete.

Theorem 2.3. Let $f$ be a decreasing, $H G$-convex (concave, respectively) function defined on $[1,+\infty)$.

(1) If $\left\{a_{n}\right\}$ an increasing, positive sequence such that $\left\{n\left(\frac{a_{n}}{a_{n+1}}-1\right)\right\}$ decreases (the sequence $\left\{n\left(\frac{a_{n+1}}{a_{n}}-1\right)\right\}$ increses, respectively), then

$$
\left(\prod_{k=1}^{n} f\left(\frac{a_{n}}{a_{k}}\right)\right)^{1 / n} \geq\left(\prod_{k=1}^{n+1} f\left(\frac{a_{n+1}}{a_{k}}\right)\right)^{1 /(n+1)} .
$$

(2) If $\varphi$ be an increasing convex positive function defined on $(0, \infty)$ such that $\left\{\varphi(k)\left(\frac{\varphi(k)}{\varphi(k+1)}-\right.\right.$ 1)\} decreases, then

$$
\left(\prod_{k=1}^{n} f\left(\frac{\varphi(n)}{\varphi(k)}\right)\right)^{1 / \varphi(n)} \geq\left(\prod_{k=1}^{n+1} f\left(\frac{\varphi(n+1)}{\varphi(k)}\right)\right)^{1 / \varphi(n+1)}
$$

Proof. The proof runs as in the proof of Theorem 2.1. Here, the increase of $\ln f(1 / x)$ is deduced from the decrease of $f$. 
Remark 2.4. In Theorem 1.1, if we replace $f$ increasing with decreasing, then the inequality (1.3) is reversed. That is

$$
\frac{1}{n} \sum_{k=1}^{n} f\left(\frac{a_{k}}{a_{n}}\right) \leq \frac{1}{n+1} \sum_{k=1}^{n+1} f\left(\frac{a_{k}}{a_{n+1}}\right) \leq \int_{0}^{1} f(x) d x
$$

Indeed, by the decrease of $f$ on $[0,1]$ we have $-f$ is increasing. Therefore, applying directly Theorem 1.1 for this function we obtain the inequality (2.7). This implies the inequality (2.1) is reversed whenever $f$ decreasing and the inequalities (2.3), (2.5) are reversed whenever $f$ increasing.

\section{Corollaries}

From these theorems, we can obtain many new inequalities related to Alzer's inequality and others or, similar inequalities to those in [3].

Corollary 3.1. Let $\varphi$ be an increasing convex positive function defined on $(0, \infty)$ such that $\left\{\varphi(k)\left(\frac{\varphi(k)}{\varphi(k+1)}-1\right)\right\}$ decreases, then

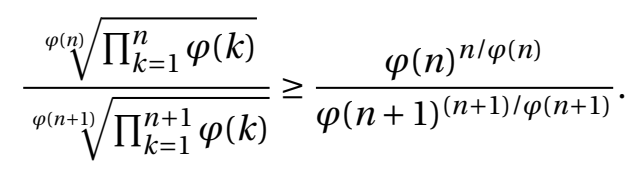

Proof. Taking $f(x)=x$ is an increasing function on $(0,1]$. Moreover, we have $\frac{f^{\prime}(x)}{f(x)}=\frac{1}{x}$ is a decreasing function on $(0,1]$. By Corollary 2.5 in [1], $f$ is $A G$-concave. So, applying Theorem 2.1 for this function we get the inequality (3.1).

Corollary 3.2. Let $r>0$ and $\varphi$ be an increasing convex positive function defined on $(0, \infty)$ such that $\left\{\varphi(k)\left(\frac{\varphi(k)}{\varphi(k+1)}-1\right)\right\}$ decreases, then

$$
\frac{1}{\varphi(n)} \sum_{k=1}^{n} \frac{\varphi(k)^{r}}{\varphi(n)^{r}} \geq \frac{1}{\varphi(n+1)} \sum_{k=1}^{n+1} \frac{\varphi(k)^{r}}{\varphi(n+1)^{r}} .
$$

Proof. Taking $f(x)=1 / x^{r}$ where $r>0$ for $x \in[1,+\infty)$. Obviously, $f$ is decreasing on $[1,+\infty)$. Moreover, we have

$$
g(x):=\left(x^{2} f^{\prime}(x)\right)^{\prime}=\left(-r x^{1-r}\right)^{\prime}=r(r-1) x^{-r}, \quad \forall x \in(1,+\infty) .
$$

It's easy to see that $g(x)>0$ whenever $r>1$ and $g(x)<0$ whenever $0<r<1$. So, by Corollary 2.5 in [1], $f$ is $H A$-convex (concave) whenever $r>1(0<r<1$, respectively). So, applying Theorem 2.2 for this function we get the inequality (3.2).

If taking $f(x)=x^{1 / x} e^{1 / x}$ for $x \in[1,+\infty)$, then $f$ is decreasing. And, we have $x^{2} f^{\prime}(x) / f(x)=$ $-\ln x$ is a decreasing function on $(1,+\infty)$. Hence, by Corollary 2.5 in [1], $f$ is $H G$-concave. By applying direct Theorem 2.3, we obtain 
Corollary 3.3. For all natural number n, the following inequality is valid

$$
\frac{n^{(n+1) / 2 n}}{(n+1)^{(n+2) / 2(n+1)}} e^{1 /[2 n(n+1)]} \geq \frac{\sqrt[n^{2}]{\prod_{k=1}^{n} k^{k}}}{\sqrt[(n+1)^{2}]{\prod_{k=1}^{n+1} k^{k}}} .
$$

\section{References}

[1] G. D. Anderson, M. K. Vamanamurthy, and M. Vuorinen, Generalized convexity and inequalities, J. Math. Anal. Appl., 335 (2007) 1294-1308.

[2] Jiding Liao and Kaizhong Guan, On Alzer's Inequality and its generalized forms, JMI, J. Math. Inequal., 4 (2010), 161-170.

[3] F. Qi and B.-N. Guo, Monotonicity of sequences involving convex function and sequence, MIA, Math. Inequal. Appl., 9 (2006), 247-254.

[4] F. Qi and B.-N. Guo, Monotonicity of sequences involving geometric means of positive sequences with monotonicity and logarithmical convexity, MIA, Math. Inequal. Appl., 9 (2006), 19.

[5] Ilhan M. Izmirli, An Elementary Proof of the Mean Inequalities, Advances in Pure Mathematics, 3(2013), 331334. http://dx.doi.org/10.4236/apm. 2013.33047.

[6] Jian-She Sun, Sequence Inequalities for the Logarithmic Convex (concave) Function, Communications in Mathematical Analysis, 1 (2006), 6-11.

[7] Liang-Cheng Wang, Monotonicity and convexity of four sequences originating from Nanson's inequalities, J. Inequal. Pure and Appl. Math., 7(2006), Art. 150.

[8] G. Zabandan, A new refinement of the Hermite - Hadamard inequality for convex functions, J. Inequal. Pure and Appl. Math., 10 (2009), Art. 45, 7 pp.

Department of Natural Science and Technology, Tay Nguyen University, Daklak, Vietnam.

E-mail: nguyenngochue2009@gmail.com

Department of Natural Science and Technology, Tay Nguyen University, Daklak, Vietnam.

E-mail: duongquochuy2009@gmail.com 